\title{
Polineuropatía motora subaguda secundaria a intoxicación por plomo
}

\author{
Subacute motor polyneuropathy secondary to \\ lead poisoning
}

\author{
Mauricio Zúñiga, María Camila Posada (Medellín), \\ Maurix Fernando Rojas, Andrés Collazos • Call (Colombia)
}

\section{Resumen}

Las manifestaciones clínicas de la intoxicación por plomo son variadas e inespecíficas y continúan siendo un reto diagnóstico, en especial cuando son producto de presentaciones tan infrecuentes como las secundarias a fragmentos por proyectiles de arma de fuego intraarticulares. En su amplio espectro de manifestaciones se han descrito cambios que van desde el déficit de atención hasta estados más avanzados como las polineuropatías. El diagnóstico se basa principalmente en la medición del metal en distintos elementos del paciente y el tratamiento en medidas generales de soporte y terapia de quelación.

Describimos el caso de un hombre con el antecedente de una herida por arma de fuego en cadera derecha, con un cuadro clínico progresivo de dolor abdominal, anemia acompañada de punteado basófilo y manifestaciones neurológicas. Con un estudio por electromiografía que reporto una polineuropatía axonal desmielinizante motora y sensitiva. Sus niveles séricos de plomo fueron 113 $\mu \mathrm{g} / \mathrm{dL}$ (Rango normal hasta $10 \mu \mathrm{g} / \mathrm{dL}$ ) y después de un esquema de tratamiento con edetato calcio disódico (EDTA) más D penicilamina los síntomas neurológicos mejoraron asociado a un control sérico de plomo de $49.1 \mu \mathrm{g} / \mathrm{dL}$.

Conclusión: la intoxicación por plomo es causa de polineuropatía motora subaguda especialmente en pacientes con exposición prolongada y niveles séricos altos de plomo. (Acta Med Colomb 2014; 39: 185-190).

Palabras clave: intoxicación por plomo, polineuropatía, terapia por quelación.

\begin{abstract}
The clinical manifestations of lead poisoning are varied and nonspecific and remain a diagnostic challenge, especially when they are product of infrequent presentations such as the secondary to intraarticular gun fire fragments. In its broad spectrum of manifestations, changes ranging from attention deficit disorder to more advanced states like polyneuropathies have been described. The diagnosis is based mainly on the measurement of metal in various elements of the patient, and treatment in general supportive measures and chelation therapy.

The case of a man with a history of gunshot wound in right hip with a progressive clinical picture of abdominal pain, anemia accompanied by basophilic stippling and neurological manifestations is described. The electromyography reported a motor and sensory axonal demyelinating polyneuropathy. Serum levels of lead were $113 \mu \mathrm{g} / \mathrm{dL}$ (normal range up $10 \mu \mathrm{g} / \mathrm{dL}$ ) and after a treatment regimen with calcium disodium edetate (EDTA ) plus D penicillamine, the neurological symptoms improved, associated with a serum lead control of $49.1 \mu \mathrm{g} / \mathrm{dL}$.
\end{abstract}

Conclusion: lead poisoning causes subacute motor polyneuropathy especially in patients with prolonged exposure and high serum lead levels. (Acta Med Colomb 2014; 39: 185-190).

Keywords: lead poisoning, polyneuropathy, chelation therapy.
Dr. Mauricio Zúñiga Luna: Especialista en Medicina Interna Universidad del Valle y Cardiología Clínica Universidad Pontificia Bolivariana. Cali, Valle; María Camila Posada: Estudiante de Medicina Universidad Pontificia Bolivariana. Medellín; Dr. Andrés Collazos: Especialista en Medicina Interna Universidad del Valle. Cali; Dr. Maurix Fernando Rojas: Especialista en Toxicología Clínica, Colegio Mayor de Nuestra Señora del Rosario. Coordinador de Toxicología, Hospital Universitario del Valle. Cali (Colombia).

Correspondencia: Dr. Mauricio Zúñiga Luna. Cali (Colombia).

E-mail: ma_zu_lu@hotmail.com Recibido: 15/I/2013 Aceptado: 04/III/2014 


\section{Introducción}

La intoxicación por plomo es más frecuente de lo que se esperaría, ya que su amplia distribución en el medio ambiente y laboral llevan a exponerse en un sinnúmero de maneras. Actualmente es frecuente su uso para manufacturas de pinturas, cosméticos, combustibles, baterías, proyectiles de arma de fuego, entre otras (1).

Sus fuentes de contaminación han sido clasificadas en ocupacional, ambienta y accidental. Un ejemplo de esta última es la presencia de fragmentos de proyectil en espacios articulares altamente vascularizados, que pueden resultar en un rápido incremento de los niveles séricos de plomo como se documenta en este caso clínico (2). El rango de referencia para definir normalidad según el centro del control y prevención de enfermedades CDC 2005 es de $5 \mu \mathrm{g} / \mathrm{dL}$ $(0.24 \mu \mathrm{mol} / \mathrm{L})$ para más de $90 \%$ de la población adulta. Los niveles de plomo en sangre (BLL) son el marcador más útil para definir intoxicación. No obstante este es un indicador de exposición reciente y no evalúa dosis acumuladas. En aras de dar solución a la disyuntiva de exposición reciente vs exposición crónica se recomienda utilizar el protoporfirin IX eritrocitario (EP), el cual puede medirse en forma libre o unida al zinc (ZPP), como un indicador de exposición en las últimas 2-6 semanas (3). Una vez absorbido $99 \%$ del plomo se une al eritrocito por aproximadamente 30-35 días y luego se redistribuye en los diferentes órganos en las siguientes 4-6 semanas (hígado, baso, cerebro, pulmones, dientes, hueso y etc). Aproximadamente $80-95 \%$ del plomo en los adultos es almacenado en los huesos, con una vida media que puede variar desde años a décadas, siendo excretado de manera continua por el riñón.
El plomo es tóxico para las mitocondrias, por consiguiente tiene un amplio espectro de manifestaciones clínicas, que guardan una relación directa con los niveles séricos (Tabla 1). Así las alteraciones hematológicas se hacen evidentes con niveles $>50 \mu \mathrm{g} / \mathrm{dL}$, producto de una disminución en la enzima pirimidina 5 nucleotidasa, que genera una alteración en la maduración de los glóbulos rojos y hemolisis temprana. Manifestándose como anemia microcítica hipocromía con niveles séricos de hierro normal, índices de reticulocitos elevados y punteado basófilo, este último puede estar presente en otro tipo de intoxicaciones como los bencenos y el arsénico (4). Las alteraciones en el sistema nervioso van desde el déficit de atención hasta estados más avanzados como las polineuropatías, que tienen diferentes modos de presentación, que dependerán del tiempo e intensidad de la exposición, pudiéndose definir una puramente motora y otra con un componente mixto.

\section{Caso clínico}

Hombre de 37 años procedente de la ciudad de Santiago de Cali, Valle del Cauca, quien consulta por un cuadro clínico de cinco años de evolución con síntomas constitucionales consistentes en pérdida de $20 \mathrm{~kg}$ de peso, fatiga fácil e irritabilidad. En los siguientes tres meses de iniciado sus síntomas comenzó con náuseas, emesis y episodios recurrentes de dolor difuso abdominal tipo cólico de fuerte intensidad refractario a analgésicos que se interpretó como un posible síndrome adherencial secundario a una laparotomía abdominal previamente realizada. En los últimos dos años se evidencia una disminución simétrica, progresiva de la fuerza en ambas manos, con una limitación para la extensión de

Tabla 1. Niveles de plomo en sangre y toxicidad.

\begin{tabular}{|c|c|c|c|c|}
\hline \multicolumn{5}{|c|}{ Nivel de plomo en sangre ug/dL (umol/L) } \\
\hline $5-9(0.24-0.43)$ & $10-19(0.48-0.92)$ & $20-39(0.97-1.88)$ & $40-79(1.93-3.81)$ & $\geq 80(\geq 3.86)$ \\
\hline $\begin{array}{l}\text { Posibles efectos adversos } \\
\text { sugeridos por estudios } \\
\text { epidemiológicos }\end{array}$ & $\begin{array}{l}\text { Posible aborto espontáneo } \\
\text { Bajo peso al nacer } \\
\text { Posibles cambios en tensión } \\
\text { arterial } \\
\text { Posible disfunción renal }\end{array}$ & $\begin{array}{l}\text { Aborto espontáneo } \\
\text { Bajo peso al nacer } \\
\text { Posibles cambios en tensión } \\
\text { arterial } \\
\text { Posible disfunción renal } \\
\text { Signos no específicos } \\
\text { - Cefalea } \\
\text { - Satiga } \\
\text { - Anorexia } \\
\text { - Constipación } \\
\text { - Diarrea } \\
\text { - Artralgias } \\
\text { - Mialgias } \\
\text { Cibido } \\
\text { Cambios de personalidad } \\
\text { Posibles efectos en SNC } \\
\text { Déficit de memoria y atención }\end{array}$ & $\begin{array}{l}\text { Aborto espontáneo } \\
\text { Bajo peso al nacer } \\
\text { Signos no específicos } \\
\text { Efectos en SNC } \\
\text { Espermatozoides } \\
\text { • Disminuidos } \\
\text { • Anormales } \\
\text { Neuropatía periférica subclínica } \\
\text { Posible HTA } \\
\text { Posible anemia } \\
\text { Posible daño renal }\end{array}$ & $\begin{array}{l}\text { Aborto espontáneo } \\
\text { Bajo peso al nacer } \\
\text { Signos no específicos } \\
\text { Efectos en SNC } \\
\text { Efectos en espermatozoides } \\
\text { Neuropatía periférica } \\
\text { HTA } \\
\text { Anemia } \\
\text { Dolor abdominal tipo cólico } \\
\text { Nefropatía }\end{array}$ \\
\hline
\end{tabular}


muñecas y dedos, que se acompañó de atrofia muscular y en el intervalo de un año progresó a incapacidad total para la extensión de ambas manos y pies hasta la postración total en cama. Como antecedentes personales tenía cuatro heridas por arma de fuego, una con proyectil subcutáneo cervical posterior derecho, la segunda en el brazo derecho con el proyectil ubicado en tejidos blandos, la tercera en la cavidad abdominal que requirió laparotomía y la cuarta en la cadera derecha con fractura asociada sin intervención quirúrgica previa, con un grado moderado de discapacidad secundaria.

Al ingreso, lucía crónicamente enfermo, pálido, caquéctico, con peso de $50 \mathrm{Kg}$, evidenciándose atrofia muscular generalizada, la cual era más evidente en el dorso de las manos y pies, sin fasciculaciones, cuadriparético de predominio distal, con una escala de fuerza muscular de $5 / 5$ con la siguiente distribución (Tabla 2), reflejos osteotendinosos disminuidos, sin nivel sensitivo, ni compromiso de esfínteres y sin signos de neurona motora superior (Figura 1).

En el hemoleucograma se evidenció anemia de volúmenes normales, por lo que se realizó un extendido en sangre periférica documentando punteado basófilo (Figura 2), su
Tabla 2. Escala de fuerza y distribución.

\begin{tabular}{|c|c|c|}
\hline Derecho & Músculos & Izquierdo \\
\hline $4 / 5$ & Deltoides-Bíceps & $4 / 5$ \\
$1 / 5$ & Extensor del carpo & $1 / 5$ \\
$4 / 5$ & Tríceps & $4 / 5$ \\
$1 / 5$ & Flexor profundo de los dedos & $1 / 5$ \\
$1 / 5$ & Interóseos & $1 / 5$ \\
$4 / 5$ & Ileopsoas & $4 / 5$ \\
$4 / 5$ & Cuádriceps & $4 / 5$ \\
$1 / 5$ & Tibial anterior & $1 / 5$ \\
$1 / 5$ & Ext largo del halux & $1 / 5$ \\
$1 / 5$ & Gastronemios & $1 / 5$ \\
\hline
\end{tabular}

índice reticulocitario era indicativo de reactividad medular con Coombs directo negativo y niveles de ferritina normal; no se hallaron alteraciones en la química sanguínea, sedimento urinario y ni radiografía de tórax. Se procedió a realizar una electromiografía con velocidad de conducción

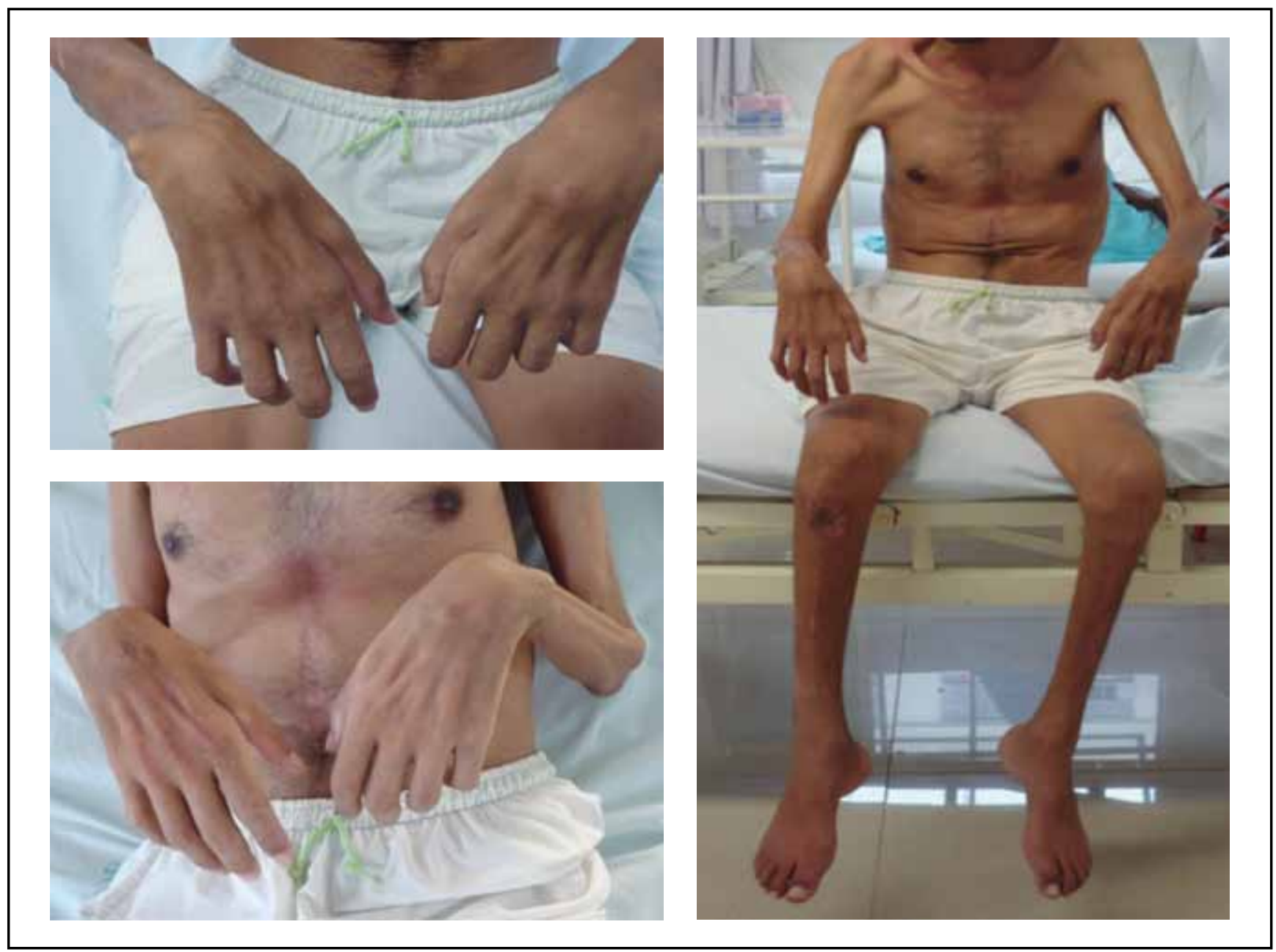

Figura 1. Examen físico. Evidencia atrofia muscular generalizada (dorso, manos y pies); cuadriparesia distal. 


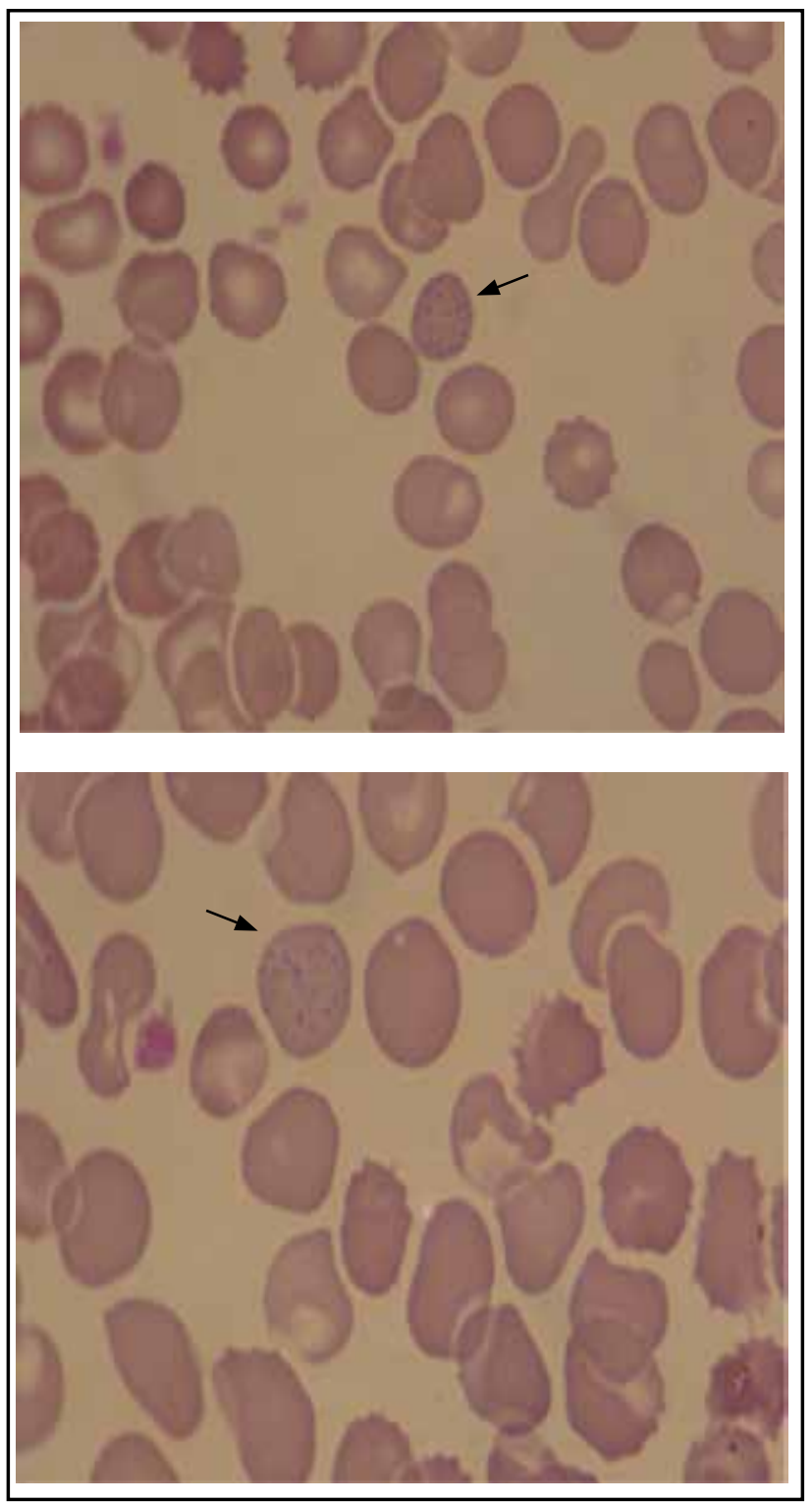

Figura 2. Extendido de sangre periférica. Punteado basófilo.

donde se documentó una polineuropatía axonal demielinizante con compromiso motor y sensitivo de predominio distal. Se hizo un análisis en el que se resaltó su compromiso simétrico distal de una polineuropatía motora axonal de lenta evolución en presencia de disestesias, por lo que se inició una búsqueda activa frente a causas etiológicas consistentes en entidades sistémicas, metabólicas y tóxicas. Se reportó un Elisa para VIH, antígenos de superficie para hepatitis $\mathrm{B}$ y anticuerpos contra la hepatitis $\mathrm{C}$ negativos $\mathrm{y}$ citoquímico de líquido cefalorraquídeo normal. Niveles de hormona estimulante de tiroides, glicemia en ayunas, porfobilinógeno en orina y vitamina B12 normal. Se realizaron niveles de antígeno carcinoembrionario, alfa feto proteína y antígeno prostático especifico normales. La electroforesis de proteínas fue reportada con niveles de albúmina,
Tabla 3. Reporte de paraclínicos

\begin{tabular}{|lc|lc|}
\hline Paraclínicos & & Paraclínicos & \\
\hline Leucocitos & $6.220 / \mathrm{mm}^{3}$ & Creatinina & $1.0 \mathrm{mg} / \mathrm{dL}$ \\
Neutrófilos & $62.5 \%$ & BUN & $28 \mathrm{mg} / \mathrm{dL}$ \\
Linfocitos & $25.9 \%$ & Sodio & $139 \mathrm{mEq} / \mathrm{L}$ \\
Hemoglobina & $7.5 \mathrm{~g} / \mathrm{dL}$ & Cloro & $113 \mathrm{mEq} / \mathrm{L}$ \\
Hematocrito & $23.8 \%$ & Calcio & $9.3 \mathrm{mEq} / \mathrm{L}$ \\
VCM & $83.1 \mathrm{fL}$ & Magnesio & $2.1 \mathrm{mEq} / \mathrm{L}$ \\
Plaquetas & $353.000 / \mathrm{uL}$ & Potasio & $4.6 \mathrm{mEq} / \mathrm{L}$ \\
Punteado basófilo & ++++ & CK total & $190 \mathrm{u} / \mathrm{L}$ \\
Ferritina & $100 \mathrm{ng} / \mathrm{mL}$ & Vitamina B12 & $488 \mathrm{pg} / \mathrm{ml}$ \\
CAE & $2.3 \mathrm{ng} / \mathrm{mL}$ & Porfobilinogeno & Negativo \\
Alfa feto proteína & $2 \mathrm{UI} / \mathrm{mL}$ & Elisa VIH & Negativo \\
Plomo & $113 \mathrm{ug} / \mathrm{dL}$ & Anas & Negativo \\
\hline
\end{tabular}

alfa 1 y 2 , beta y gama normales (Tabla 3 ). La radiografía de cadera derecha evidenció un proceso osteoartrítico con esquirlas de proyectil intraarticulares e infiltración extensa de tejidos blandos adyacentes (Figura 3), a pesar de no tener antecedentes de exposición laboral relevantes y haciendo énfasis en la presencia de fragmentos de proyectil por arma de fuego en cadera derecha, se realizaron niveles séricos de plomo, en búsqueda de una intoxicación crónica secundaria, con reporte de $113 \mu \mathrm{g} / \mathrm{dL}(\mathrm{Nr}<10 \mu \mathrm{g} / \mathrm{dL})$, confirmando la sospecha diagnóstica, por lo que se procedió a realizar terapia de quelación con edetato calcio disodico (EDTA) a $20 \%$ a un dosis de $30 \mathrm{mg} / \mathrm{kg}$ día por cinco días y se continuó con D penicilamina $250 \mathrm{mg}$ cada ocho horas por 20 días, con un control sérico en 30 días que se reportó de $49.1 \mu \mathrm{g} / \mathrm{dL}(\mathrm{Nr}<10 \mu \mathrm{g} / \mathrm{dL})$. Se solicitó concepto por el servicio de ortopedia definiendo plan quirúrgico consistente en desbridamiento, curetaje extenso de tejidos blandos más remplazo total de cadera y aloinjerto óseo, el cual se realizó sin complicaciones .

El paciente fue dado de alta 55 días después de haber ingresado, con una mejoría parcial de su déficit neurológico, sin episodios de dolor abdominal, resolución de su síndrome anémico y plan de rehabilitación física.

\section{Discusión}

A pesar de la alta exposición ambiental por plomo, las neuropatías son cada vez más infrecuentes. Reportándose en un seguimiento de 40 años en un centro de referencia como la Clínica Mayo no más de 10 casos, de 3 1/2 millones de pacientes evaluados (5). Varias hipótesis se han propuesto para explicar el efecto que lleva a neuropatía, sugiriendo un mecanismo de neurotoxicidad por alteración de la barrera hematoneuronal que produce edema endoneural con lesión directamente sobre la célula de Schwann o interfiriendo con la microcirculación para producir isquemia y degeneración; no obstante la teoría más aceptada es un desorden en el metabolismo de la biosíntesis 

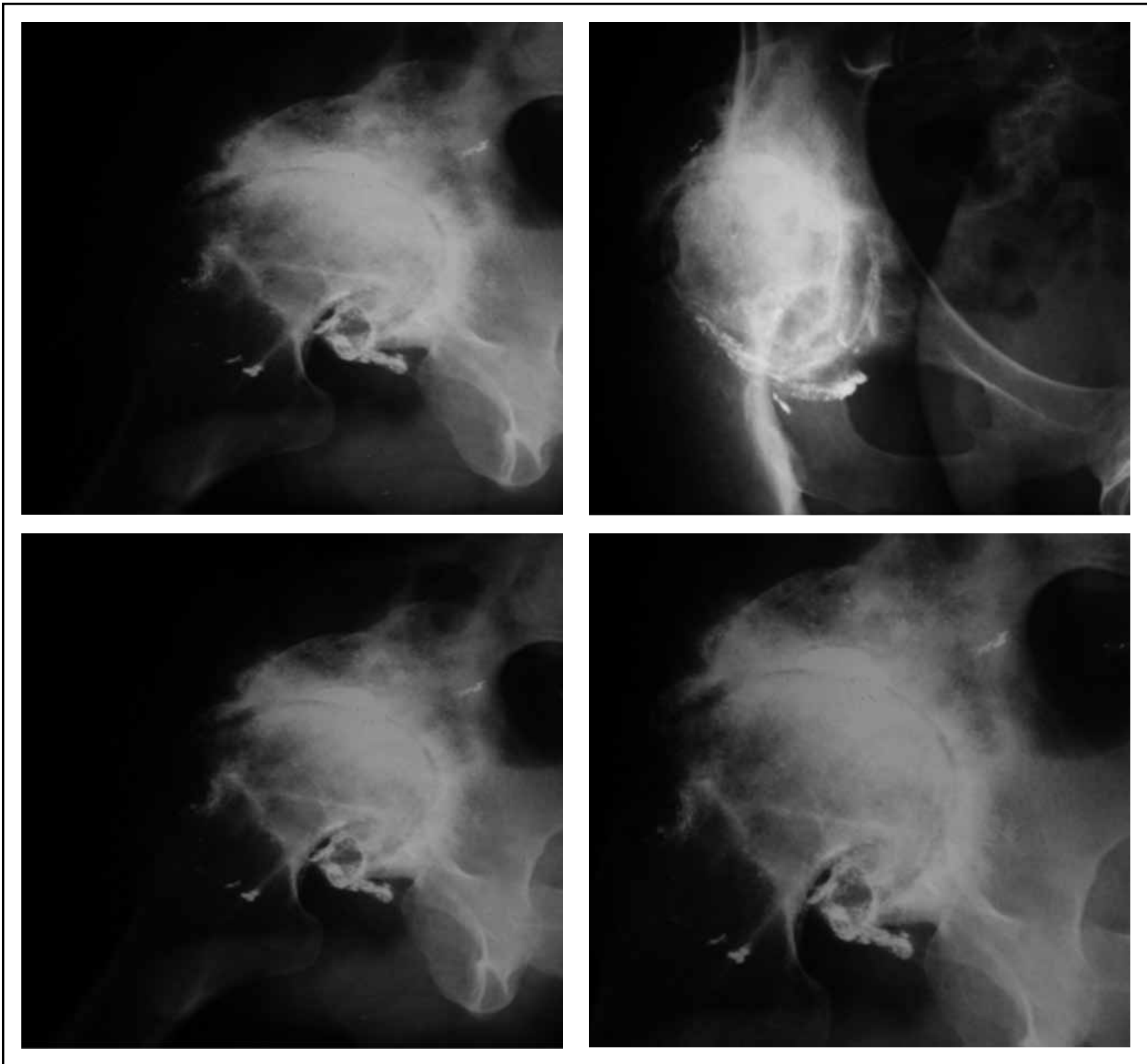

Figura 3. Radiografía de cadera. Proceso osteoartrítico con esquirlas de proyectil intraarciculares.

de grupo hemo, producto de la inhibición de las enzimas ferrochelatasa y delta aminolevulinicodehidratasa que lleva a la acumulación de metabolitos intermedios como el porfobilinógeno (6).

Las manifestaciones clínicas de las neuropatías por plomo dependerán de la duración e intensidad de la exposición, describiéndose dos tipos; la comúnmente descrita como clásica, que es única entre las polineuropatías, producto de una exposición subaguda en promedio de cinco años $\mathrm{y}$ a altas concentraciones, que tempranamente produce un compromiso puramente motor y severo sobre los músculos extensores de las muñecas y de los dedos antes que cualquier otro grupo muscular, llevando a debilidad sin fasciculaciones. El compromiso sobre los músculos tenares e interóseos es infrecuente; de igual modo hay debilidad en extremidades distales de miembros inferiores confinada a músculos dorsiflexores y extensores de los dedos; raramente hay compromiso proximal y ocasionalmente en intoxicaciones severas se han descritos caso de cuadriparesia, debilidad en músculos de cuello y extraoculares (7). Aunque infrecuentes las alteraciones sensitivas pueden estar presentes. El pronóstico es bueno entre más temprano se halla retirado el paciente de la exposición, presentando recuperación lenta e incompleta. Existe una similitud entre las manifestaciones de la neuropatía reportada en los pacientes con porfiria y la subaguda por intoxicación por plomo, así que no es infrecuente encontrar niveles elevados de ácido delta aminolevulénico y coproporfirinas (8). 
En este caso a pesar de múltiples muestras, los niveles de porfobilinógeno en orina fueron normales.

El segundo tipo de neuropatía que frecuentemente se ha descrito en pacientes con intoxicación por plomo es producto de una exposición prolongada, en promedio de 10-15 años y en concentraciones bajas, definiendo un componente de polineuropatía mixta de predominio sensitivo, distal y simétrico acompañado de síntomas autonómicos. Que puede aparecer entre los siete y 45 años posteriores a la exposición, con un pronóstico que parece ser más incierto (9). Esta última es la que frecuentemente ha sido reportada en pacientes con fragmentos de bala retenidos, no obstante en nuestro caso, las manifestaciones clínicas son las de una polineuropatía subaguda, posiblemente explicada por el alojamiento del proyectil en un área altamente vascularizada que llevó a unos niveles séricos de plomo muy por encima de los que se han reportado en los pacientes con cuadro de polineuropatía mixta.

Las estrategias de tratamiento están indicadas en pacientes con niveles séricos elevados de plomo y en los que hay evidencia de disfunción de órganos (Tabla 4). Aunque en la práctica clínica no hay recomendación alguna para la extracción de proyectiles por arma de fuego, la presencia de éstos en áreas de gran vascularización y absorción como son el espacio articular, el tracto gastrointestinal, el estrecho contacto con LCR, al igual que la fragmentación de los mismos en presencia de fracturas óseas, deberán llevar a monitorizar los niveles séricos de plomo y evaluar la posibilidad de extraerlos (10). La FDA ha aprobado dos tipos de agentes quelantes, el EDTA y DMSA, sin poder definir la superioridad de uno frente al otro, ya que no se cuenta con estudios clínicos comparativos. La D penicilamina es igualmente efectiva en aumentar la excreción renal de plomo (11).

Este es el primer caso reportado en nuestro hospital. Y aunque infrecuente deberá ser incluido en el diagnóstico
Tabla 4. Recomendaciones de terapia de quelación en intoxicación por plomo.

\begin{tabular}{|l|c|}
\hline Recomendación & Niveles séricos de plomo \\
\hline La terapia es recomendada para todos los adultos & $>100 \mu \mathrm{g} / \mathrm{dL}$. \\
La terapia puede ser fuertemente considerada & $80-99 \mu \mathrm{g} / \mathrm{dL}$ \\
La terapia en presencia de síntomas es & $50-79 \mu \mathrm{g} / \mathrm{dL}$ \\
posiblemente considerada & \\
\hline
\end{tabular}

diferencial de otras afecciones posiblemente más comunes en nuestro medio, como las polineuropatías metabólicas y las asociadas a enfermedades sistémicas, que también tienen una presentación subaguda, con componente motor.

\section{Referencias}

1. Wedeen R, Goldman R, Headapohl D, Hipkins K, Hu H, Kosnett M, et al. Medical Management Guidelines for Lead Exposed Adults. MMWR 2007.

2. Coon T, Miller M, Shirazi F, Sullivan J. Lead Toxicity in a 14-year-old Females With Retained Bullet Fragments. Pediatrics 2006; 117: 227-230.

3. Zura R, Bosse M. Curren Treatment of gunshot Wounds to the Hip and Pelvis. Clinical Orthopaedics and Research. 2003; 408: 410-414.

4. Philip AT, Gerson B. Lead Poisoning - Part II. Effects and Assay. Clin Lab Med 1994; 14: 651-670.

5. Dyck P, Thomas P, Griffin J, Low P, Poduslo J. Peripheral Neuropathy Philadelphia Wb saunder 1993; 1549-1570.

6. Ohnishi A, Schilling K, Brimijoin W, Lambert E, Fairbanks V, Dyck P. Morphometry, nerve conduction, and choline acetyltransferase transport; new findin of edema associated with segmental demylination. J Neuropathol Exp Neurl 1997; 36: 499-518.

7. Occupational Safety and Health Administration.http://aoec.org/documents/ positions/MMG_FINAL.pdf.

8. Rubens O, Logina I, Kravel I, Eglite M, Donaghy M. Peripheral neuropathy in chronic occupational inorganic lead exposure: a clinical and electrophysiological study. J Neurol Nerosurg Psychiatry 2001; 71: 200-204.

9. Thomson R, Parry G. Neuropathies Associated With Excessive Exposure to Lead. MuscleNerve 2006; 33: 732-741.

10. McQuirter JL, Rothenberg SJ, Dinkins GA, Manalo M, Kon-drashov V, Todd AC. The effects of retained lead bullets on body lead burden. J Trauma 2011; 50: 892-899.

11. Marcus S. Experience with D penicillamine in Treating lead poisoning. Vet Hum Toxicol 1982; 24:18-20. 